\title{
What is Dysbiosis and, in which Parts of the Organism Exists?
}

\section{ISSN: 2637-7632}

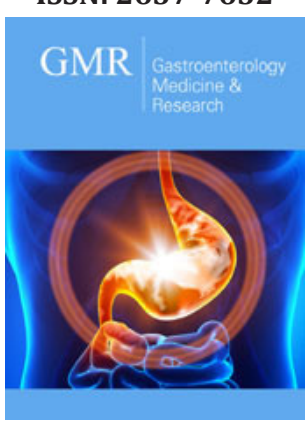

*Corresponding author: Álvaro Zamudio Tiburcio, Department of Gastroenterology, Intestinal Microbiota Transplantation Hospital Trinidad, Mexico

Submission: 悳 October 29, 2019

Published: 倹December 04, 2019

Volume 4 - Issue 1

How to cite this article: Álvaro Z T, Héctor B R, Pedro A R L, María MA G, Nydia Á V. What is Dysbiosis and, in which Parts of the Organism Exists?. Gastro Med Res. 4(1). GMR.000580. 2019.

DOI: $10.31031 /$ GMR.2019.04.000580

Copyright@ Álvaro Zamudio Tiburcio, This article is distributed under the terms of the Creative Commons Attribution 4.0 International License, which permits unrestricted use and redistribution provided that the original author and source are credited.

\author{
Álvaro Zamudio Tiburcio ${ }^{1 *}$, Héctor Bermúdez Ruiz ${ }^{2}$, Pedro Antonio Reyes \\ López ${ }^{3}$, María Magdalena Aguirre García ${ }^{4}$ and Nydia Ávilla Vanzzinin ${ }^{5}$ \\ ${ }^{1}$ Department of Gastroenterology, Mexico \\ ${ }^{2}$ Department of Endoscopy, Mexico \\ ${ }^{3}$ Department of Cardiology, Mexico \\ ${ }^{4}$ Head of the Faculty section of Medicine in Research, Mexico \\ ${ }^{5}$ Department of External Consultation, Mexico
}

\begin{abstract}
A thorough review of how microbial dysbiosis of different regions impacts on health. The dysbiosis of the Intestinal Microbiota stands out, since it is the one that occupies the highest percentage and, where the greatest number of investigations have been carried out. The different axes that have sprung from the studies are determined, highlighting the brain-gut microbiota axis. The various bi-directional communications between the microbiota and the different organs are analyzed and as this communication is significant in the loss of health.
\end{abstract}

Keywords: Dysbiosis; Dysbacteriosis; Gut-microbiota-brain axis (GMB); Intestinal Microbiota Transplantation (IMT); Intestinal Microbiota (IM)

\section{Comments}

Dysbiosis (dysbacteriosis). "Microbiota imbalance that can be caused by various etiologies or mismatch thereof, which results in multiple alterations and, consequently in diseases." This imbalance produces inflammation in various organs and systems, such as the liver [1], the intestine; by causing Inflammatory Bowel Disease [2], the stomach [3], other autoimmune processes or tumor conditions [4]. The skin is not exempt [5], through communication with the Microbiota, through the Intestine-Skin Axis. As well as the neuro-psychiatric problems, mediated by the Intestine-Microbiota-Brain Axis [6]. Now, how can it be done so that the Intestinal Microbiota does not reach dysbiosis? [7] First of all, we must try to preserve its homeostasis; that is, try to maintain those favorable microorganisms, through healthy diets [8], strict control of the use of antibiotics [9] and bacterial intervention [10]. The use of probiotics has been discussed in the search for homeostasis maintenance, some authors [11] point out that they can restore MI; Lactobacillus being the most indicated. Other authors $[12,13]$ include, in addition to Lactobacillus spp., Bifidobacterium spp., And Saccharomyces spp. In addition to the use of symbiotics. In what regions of the body are there dysbiosis. The answer is that in anyone, since the microbiota is present in practically the whole organism, although in smaller quantity, but with the same functions as the digestive one. IM is one of the most studied subjects, especially that which occurs due to the infection of Clostridium difficile. Topic that is not even under discussion, since Microbiota Transplantation is highly effective in these processes. Irritable Bowel Syndrome (IBS) and other functional digestive disorders follow. Inflammatory Bowel Disease, preferably nonspecific chronic Ulcerative Colitis (UC), and different diarrhea [14-17].

Dysbiosis in Oncology. Many Oncologists have turned their eyes to oncological processes and their relationship with dysbiosis. Among the most studied and improved cancers with IM, there are those of the colon, gastric and gallbladder [18]. Dysbiosis of the skin. More often we will hear about the different axes that make up the relationship between the Microbiome and parallel systems, such as the Intestine-Microbiota-Brain axis, the intestine-skin axis, the intestine-lung axis, etc. [19] These axes, where bidirectional communication is so effective 
and, in the case of the skin, carry signals that involve the most extensive organ of the human body and, we see conditions such as Atopic Dermatitis appear [20]. Alterations of the skin microbiota have been observed in Psoriasis, [21] including Vitiligo, that is, dysbiosis is present [22]. The aforementioned processes, although they improve with Intestinal Microbiota Transplantation, are not fully resolved. Could a skin-skin transplant be required? But what happens with acne? a condition so widespread throughout the world. It has been observed that the Intestinal Microbiota is different and it produces dysbiosis as a cause of the disease [23]. Finally, another frequent condition such as Eczema, has been widely discussed and at the moment the comments focus on the language used [24]. Dysbiosis in ears, nose and throat. This region is not exempt from the link between the Microbiota in the area [25] and the diseases, especially chronic diseases that occur in it, through intestinal dysbiosis and the indicated region [26].

Dysbiosis in the airway. What's new about it? Dysbiosis of the intestinal microbiota has been implicated in conditions such as asthma, allergies and cystic fibrosis [27]. It has also been linked to changes in the immune response and the occurrence of lung diseases. So, the use of the Intestinal Microbiota in Lung Diseases is hopeful [28]. Dysbiosis in Neuropsychiatry. Although in the neuropsychiatric processes the dysbiosis of the Intestinal microbiota has been taken as a basis, also the microbiota regulated by the intestine-microbiota-brain axis, is of fundamental importance [29]. Numerous are the conditions that occur due to this inflammation such as Autism [30], Major Depression [31], Alzheimer's [32], Multiple Sclerosis [33], Chronic Fatigue Syndrome [34], Anxiety [35], etc.

\section{Dysbiosis in endocrinological processes. What is there about} it?

Several articles highlight the relationship between Type 1 Diabetes Mellitus and dysbiosis. Among them we have those reported by Zheng P and his group [36], where through thorough analysis, they describe the interesting link between dysbiosis and Type 1 Diabetes Mellitus. The above has also been analyzed by other authors [37,38]. Something similar occurs in Hashimoto's Disease [39], where there is improvement of dysbiosis, when using the great benefits of Intestinal Microbiota Transplantation (IMT). The articles that link dysbiosis with nonalcoholic fatty liver and its improvement with Intestinal Microbiota are not negligible [40]. As well as those who refer to the metabolic syndrome [41]. One of the most frequent conditions in the world is obesity, a disorder in which numerous and interesting treatment schemes have been sought, both medical and surgical. Now, how does dysbiosis influence these processes and, is the intestinal microbiota considered useful in them? Up to $66 \%$ of dysbiosis has been found in obese patients [42] and it has been determined that intestinal dysbiosis substantially influences the condition [43]. IMT has been shown to be effective in treating Morbid Obesity, since Obesity is a state of continuous inflammation [44].

Dysbiosis in Rheumatic Diseases. We will review two of the most common as are Osteoarthritis and Osteoarthritis. Regarding Osteoarthritis, a new metabolic phenotype has been detected, even though the mechanisms of how these metabolites of the Intestinal Microbiota contribute in their pathogenesis are not yet clear [45]. Regarding the effects of Intestinal Microbiota Transplantation in this condition, its possible effect on the condition has been considered [46]. Osteoarthritis, as another process with diverse inflammation, which in many cases is so intense that it produces disability, has been included among the conditions that have to do with dysbiosis. And not only intestinal, but dermal, oral and pulmonary [47]. Dysbiosis of the oral Microbiota. Oral microorganisms can move to the intestine and cause dysbiosis of the microbiota located there [48].

It has been shown that Porphyromonas gingival is can be transferred to the intestine [49]. These and other analyzes [50] conclude the importance of the microbiota, as a whole, in these frequent conditions. Dysbiosis in the Urinary Microbiota. Now, are prostate and bladder related to intestinal dysbiosis? It is definitely also involved. For example, the link between prostate cancer and dysbiosis has been reported [51]. This pathology also includes the urinary microbiota. Vaginal dysbiosis. There is the same inflammatory mechanism, already considered, between vaginal candidiasis and bacterial vaginosis [52]. Ocular dysbiosis. Dysbiosis in other regions affects eye health [53]. It is therefore advisable to keep these microorganisms healthy. Dysbiosis in immunological processes. We have left this topic to the last, considering it the most important. Numerous diseases of the immune system due to dysbiosis have also been reported. Among them are Intestinal Inflammatory Disease [54], Systemic Lupus Erythematosus [55], Rheumatoid Arthritis [56], fibromyalgia [57], Refractory Pouchitis [58], Idiopathic thrombocytopenic purpura [59] and fatigue syndrome chronicle [60]. All of them with good to extraordinary results, when using the Intestinal Microbiota as therapy.

\section{Conclusion}

A. Is the Intestinal Microbiota the best non-steroidal antiinflammatory that exists?

B. Are there many axes in the various systems as there are communities of existing microorganisms?

C. The IMT is beneficial in many pathologies?

D. In the near future, all the work will be normalized, not only of the intestinal microbiota, but of all the microbiota.

Conflicts of Interest: The authors declare that they do NOT have affiliation or participation in organizations with financial interests.

Ethical Approval: This report does not contain any study with human or animal subjects carried out by the authors.

Informed Consent: The authors obtained informed written consent from the patients, in order to develop this article.

\section{References}

1. Saltzman ET, Palacios T, Thomsen M, Vitetta L (2018) Intestinal microbiome shifts, dysbiosis, inflammation, and non-alcoholic fatty liver disease. Front Microbiol 9: 61. 
2. Tamboli CP, Neut C, Desreumax P, Colombel JF (2004) Dysbiosis in in flammatory bowel disease. BMJ Gut 53(1): 1-4.

3. Toor D, Wsson MK, Kumar P, Karthikeyan G, Kaushik NK, et al. (2019) Dysbiosis disrupts gut immune homeostasis and promotes gastric diseases. Int J Mol Sci 20(10): 2432.

4. Rea D, Coppola G, Palma G, Barbieri A, Luciano A, et al. (2018) Microbiota effects on cancer: From risks to therapies. Oncotarget 9 (25): 1791517927.

5. Salem I, Ramser A, Isham N, Ghannoum MA (2018) The gut microbiome as a major regulator of the gut-skin axis. Front Microbiol 9: 1459.

6. Lucas G (2018) Gut thinking: The gut microbiome and mental health beyond the head. Microb Ecol Health 29 (2).

7. Carding S, Verbeke K, Vipond DT, Corfe BM, Owen LJ, et al. (2015) Dysbiosis of the gut microbiota in disease. Microb Ecol Health Dis 26: 26191.

8. Conlon MA, Bird AR (2015) The impact of diet and lifestyle on gut microbiota and human health. Nutrients 7(1): 17-44.

9. Yoon MY, Yoon SS (2018) Disruption of the gut ecosystem by antibiotics. Yonsei Med J 59(1): 4-12.

10. Gagliardi A, Totino V, Cacciotti F, Iebba V, Neroni B, et al. (2018) Rebuilding the gut microbiota ecosystem. Int J Environ Res Public Health 15(8): 1679.

11. Hemarajata P, Versalovic J (2012) Effects of probiotics on gut microbiota: Mechanism of intestinal immunomodulation and neuromodulation. Therap Adv Gastroenterol 61(1): 39-51.

12. Azad AK, Sarker M, Li T, Yin J (2018) Probiotics species in the modulation of gut microbiota: An overview. Biomed Res Int pp. 1-8.

13. Mouw M (2019) The effects of probiotics and prebiotics in our gut microbiota. Gut Microbiota for Health.

14. Bien J, Palagani V, Bozko P (2012) The intestinal microbiota dysbiosis and Clostridium difficile infection: Is there a relationship with inflammatory bowel disease? Therap Adv Gastroenterol 6(1): 53-68.

15. Janeiro RBK, Vicario M, Cotoner AC, Pascua GR, Santos J, et al. (2018) A review of microbiota and irritable bowel syndrome: Future in therapies. Adv Ther 35(3): 289-310.

16. Mazurak EP (2018) Dysbiosis in functional bowel disorders. An Nutr Metab 72(4): 296-306.

17. Sorrentino D. Microbial dysbiosis in spouses of ulcerative colitis patients: Any clues to disease pathogenesis. World J Gastroenterol 23(37): 6747-6749.

18. Weng MT, Chiu YT, Wei PY, Chiang CW, Fang HL, et al. (2019) Microbiota and gastrointestinal cancer. J Formosal Med Assoc 118(Suppl 1) S32-S41.

19. Da Silva T, Dos Santos A (2013) Intestinal microbiota; Relevance to obesity and modulation by prebiotics and probiotics. Nutr Hosp 28 (4): 1039-1048.

20. Lee SY, Lee E, Park YM, Hong SJ (2018) Microbiome in the gut-skin axis in atopic dermatitis. AAIR 10(4): 354-362.

21. Visser MJE, Kell DB, Pretorius E (2019) Bacterial dysbiosis and translocation in psoriasis vulgaris. Front Cell Infect Microbiol 9: 7.

22. Ganju P, Nagpal S, Mohammed MH, Kumar PN, Pandey R, et al. (2016) Microbial communty profiling shows dysbiosis in the lesional skin of Vitiligo subjects. Scientific Reports.

23. Deng Y, Wang H, Zhou J, Mou Y, Wang G, et al. (2018) Patients with acne vulgaris have a distinct gut microbiota in comparison with healthy control. Acta Derm Venereol 98(8): 783-790.

24. Myles IA (2019) Allergy as a disease of dysbiosis: Is it time to shift the treatment paradigm? Front Cell Infect Microbiol 9: 50
25. Lee K, Pletcher SD, Lynch SV, Goldberg AN, Cope AK (2018) Heterogenity of microbiota dysbiosis in chronic rhinosinusitis: potential clinical implications and microbial community mechanisms contributing to sinonasal inflammation. Front Cell Infect Microbiol 8: 168.

26. Copeland E, Leonard K, Carney R, Kong J, Forer M, et al. (2018) Chronic rhinosinusitis: Potential role of microbial dysbiosis and recommendations for sampling sites. Front Cell Infect Microbiol 8: 57.

27. Anand S, Mande SM (2018) Diet, microbiota and gut-lung connection. Front Microbiol 9: 2147.

28. Budden KF, Gellatly SL, Wood DL, Cooper MA, Morrison M, et al. (2017) Emerging pathogenic links between microbiota and the gut-lung axis. Nat Rev Microbiol 15(1): 55-63.

29. Rogers GB, Keating DJ, Young RL, Wong ML, Wesselingh S, et al. (2016) From gut dysbiosis to altered brain function and mental illness; mechanisms and pathways. Mol Psychiatry 21(6): 738-748.

30. Hughes HK, Rose D, Ashwood P (2018) The gut microbioma and dysbiosis in autism spectrum disorders. Curr Neurol Neurosci Rep 18(11): 81.

31. Mann J, Miller JM, Sublette ME, Uhlemann AC, Cheung SG, et al. (2019) Systematic review of gut microbiota and major depression. Front Psychiatry 10: 34 .

32. Jiang C, Li G, Huang P, Liu Z, Zhao B, et al. (2017) The gut microbiota and alzheimer disease. J Alzheiemers Dis 58(1): 1-15.

33. Kirby TO, Ochoa RJ (The gut microbiome in multiple sclerosis: A potential therapeutic avenue Med Sci 6(3): 69.

34. Du Preez S, Corbitt M, Cabanas H, Eaton N, Staines D, et al. (2018) A systematic review of enteric dysbiosis in chronic fatigue syndrome/myalgic encephalomyelitis. Syst Rev 7(1): 241.

35. Cheung SG, Goldenthal AR, Uhlemann AC, Mann JJ, Miller JM, et al. (2019) systematic review of gut microbiota and major

depression. Front Psychiatry 10:34.

36. Zheng P, Li Z, Zhou Z (2018) Gut microbiome in type 1 diabetes: A comprehensive review. Diabetes Metab Res Rev 34(7): e3043.

37. Han H, Li Y, Fang J, Liu G, Yin J, et al. (2018) Gut microbiota and type 1 diabetes. Int J Mol Sci 19(4): 995.

38. Absellatif AM, Sarvetnick NA (2019) Current understanding of the role of gut dysbiosis in type 1 diabetes. J of Diabetes 11(12): 632-644.

39. Virili C, Fallahi P, Antonelli A, Benvenga S, Centanni M, et al. (2018) Gut microbiota and hashimoto's thyroiditis. Rev Endocr Metab Disord 19(4): 293-300.

40. Gérard P, Safari Z (2019) The links between the gut microbiome and non-alcoholic fatty liver disease (NAFLD) Cell Mol Life Sci 76(8): 15411558

41. Belizário JE, Faintuch J, Garay MM (2018) Gut microbiome dysbiosis and immunometabolism: New frontiers for treatment of metabolic diseases. Meditors Inflammation p. 12.

42. Farup PG, Aasbrenn M, Valeur J (2018) Separating "good" from "bad" faecal dysbiosis-evidence from two cross-sectional studies. BMC Obes.

43. Muscogiuri G, Cantone E, Cassarano S, Tuccinardi D, Barrea L, et al. (2019) Gut microbiota: A new path to treat obesity. Int J Obesity Suppl 9(1): 10-19.

44. Jayasinghe TN, Chiavaroli V, Holland DJ, Cutfield WS, O'Sullivan JM, et al. (2016) The new era of treatment for obesity and metabolic disorders: Evidence and expectations for gut microbiome transplantation. Front Cell Infect Microbiol.

45. Szychlinska MA, Di Rosa M, Castorina A, Mobasheri A, Musumeci G, et al (2019) A correlation between intestinal microbiota dysbosis and osteoarthritis. Heliyon 5(1): e01134. 
46. Li Y, Luo W, Deng Z, Lei G (2016) Diet-intestinal microbiota axis in osteoarthritis: A possible role. Mediators pp.1-4.

47. Rosenbaum JT, Asquith MJ (2016) The microbiome: A revolution in treatment for rheumatic diseases? Curr Rheumatol Rep 18(10): 62.

48. Olsen I, Yamazaki K (2019) Can oral bacteria affect the microbiome of the gut? J Oral Microbiol 11(1): 1586422.

49. Olsen I, Lambris JD, Hajishengallis G (2017) Porphyromonas gingivalis disturbs host-commensal homeostasis by changing complement function. J Oral Microbiol 9(1): 1340085.

50. Atkinson TP, Centor RM, Li X, Wang F, Van Der Pol W, et al. (2018) Analysis of the tonsilar microbiome in young adult with sore throat reveals a high relative abundance of fusobacterium necrophrum with low diversity. PLoS One 13(1): e0189423.

51. Massari F, Mollica V, Di Nunno V, Gatto L, Santoni M, et al. (2019) The human microbiota and prostate cancer: Friend or foe? Cancers 11(4): 459.

52. Pramanick R, Mayadeo N, Warke H, Begum S, Aich P, et al. (2019) Vaginal microbiota of a symptomatic bacterial vaginosis and vulvovaginal candidiasis: Are they different from normal microbiota? Microb Pathog 134: 103599.

53. Kugadas A, Gadjeva M (2016) Impact of microbiome on ocular health. Ocult Surf 14(3): 342-349.
54. DeGruttola AK, Low D, Mizoguchi A, Mizoguchi E (2016) Current understanding of dysbiosis in disease in human and animal models. Inflamm Bowel Dis 22(5): 1137-1150.

55. Rahbar Saadat Y, Hejazian M, Bastami M, Hosseinian Khatibi SM, Ardalan M, et al. (2019) The role of microbiota in the pathogenesis of lupus: Dose it impact lupus nephritis? Pharmacol Res 139: 191-198.

56. Picchianti DA, Panebianco C, Salemi S, Sorgi ML, Di Rosa R, et al. (2018) Analysis of gut microbioma in rheumatoid arthritis patients: disease-related dysbiosis and modifications induced by etanercept. Int J Mol Sci 19(10): 2938.

57. Minerbi A, Gonzalez E, Bereton NJB, Anjarkouchian A, Dewar K, et al. (2019) Altered microbiome composition in individual with fibromyalgia. Pain 160(11):2589-2602.

58. Fang S, Kraft CS, Dhere T, Srinivasan J, Begley B, et al. (2016) Successful treatment of chronic Pouchitis utilizing fecal microbiota transplantation (FMT): A case report. Int J Colorectal Dis 31(5): 1093-1094.

59. Clinical trail of gut microbiota in the management of immune thrombocytopenia. US National Library of Medicine, Maryland, USA.

60. Wallis A, Ball M, Butt H, Lewis DP, McKechnie S, et al. (2018) Open-label pilot for treatment targeting gut dysbiosis in myalgic encephalomyelitis/ chronic fatigue Syndrome: Neuropsychological symponts and sex comparisons. J Transl Med 16(1): 24. 